\section{P30 PRESCRIBING VEDOLIZUMAB IN PAEDIATRIC PATIENTS WITH CROHN'S DISEASE (CD) AND ULCERATIVE COLITIS (UC) IN ACCORDANCE WITH THE CLINICAL GUIDELINE IN AN NHS TRUST}

Eimear Mc Geehan*. University Hospitals Bristol NHS Foundation Trust

\subsection{6/archdischild-2020-NPPG.39}

Introduction Vedolizumab is a gut-selective immunosuppressive biologic used to treat moderate to severe Crohn's disease and ulcerative colitis. Vedolizumab is not licensed for use in children under 18 years of age. However, there is experience of using Vedolizumab in paediatric ${ }^{1}$ patients in practice. Vedolizumab incurs a significant cost to the NHS, as a result identification of patient response to treatment is fundamental.

Aim To ensure Vedolizumab is prescribed in adherence to guidance documented in the clinical guideline 'Vedolizumab treatment for inflammatory bowel disease patients' at an NHS trust.

\section{Standards}

- $100 \%$ of patients on Vedolizumab have been diagnosed with moderate to severe ulcerative colitis or Crohn's disease

- $100 \%$ of patients on Vedolizumab have had and inadequate response with, lost response to, or are intolerant to either conventional therapy or anti-TNF treatment

- $100 \%$ of patients were assessed for risk of TB prior to starting treatment

- $100 \%$ of patient's immunisation status was reviewed prior to starting therapy

- $100 \%$ of patients were prescribed doses recommended in the guideline

- $100 \%$ of patients had Vedolizumab checklist completed:

- Prior to infusion

- During infusion

- Post infusion

- $100 \%$ of patients had outcome of disease reviewed at 3 months

Method A retrospective study was undertaken to investigate the prescribing of Vedolizumab in CD and UC patients since the implementation of the clinical guideline in June 2018. Ethics approval was not required. List of patients prescribed Vedolizumab was obtained from pharmacy dispensing system. Infusion details were obtained from patient notes. Qualitative data was obtained and analysed.

Results Four patients were prescribed Vedolizumab at the NHS trust between June 2018 and June 2019. One patient had to be excluded from the audit as they had already commenced Vedolizumab prior to the implementation of the clinical guideline. 6 out of the 7 standards were met with a result of $100 \%$ except standard 6 which was met with a result of $33 \%$ (1 out of the 3 patients).

Discussion and Conclusion 6 out of the 7 standards were completely met; highlighting a robust system in place. The publication of the guideline has ensured that vedolizumab prescribing is appropriate, as inefficient prescribing could result in treatment failure, drug wastage and safety concerns. The only standard not completely met was standard 6 , due to the use of surgical booklets on the outpatient wards, the standardised Vedolizumab checklists were not complete.

Recommendations Ensure Vedolizumab checklist is printed out from the clinical guideline and attached in the patient notes. Medical pharmacist to re-audit data yearly to ensure the clinical guideline is being adhered to.

\section{REFERENCES}

1. Entyvio, Vedolizumab. Summary of Product Characteristics, Accessed via http:// emc.medicines.org.uk Last updated 20.08.2015 [accessed 07/06/19]

2. Vedolizumab (review) for the treating moderately to severely active ulcerative colitis. June 2015, NICE Technology Appraisal Guidance 342.

3. O Ledder, A Assa, A Levine, et al. Vedolizumab in Paediatric Inflammatory Bowel Disease: A Retrospective Multi-Centre Experience From the Paediatric IBD Porto Group of ESPGHAN. Journal of Crohn's and Colitis. 2017:11;1230-1237.

\section{P31 IMPROVING PARACETAMOL SAFETY IN A TERTIARY PAEDIATRIC HOSPITAL}

${ }^{1}$ Joe McCann*, ${ }^{1}$ Rachel Copeland, ${ }^{1}$ Aideen Keaney, ${ }^{2}$ Leanne Laverty, ${ }^{1}$ Simon Marcus. ${ }^{1}$ Royal Belfast Hospital for Sick Children; ${ }^{2}$ Altnagelvin Area Hospital

\subsection{6/archdischild-2020-NPPG.40}

Aims Medication errors are perhaps the most common threat to patient safety. ${ }^{1}$ Children are particularly at risk because of variations in age and weight necessitating individual dose calculation for each patient; doses that are ten times the correct amount are occasionally given and can be life-threatening. ${ }^{2}$ Since 2015 the hospital has been seeking, as part of a Regional Quality initiative, to improve safety related to paracetamol.

Methods Previous work involved clarification of IV paracetamol dosing for neonates and children under $10 \mathrm{~kg}$ with procurement of $100 \mathrm{mg}$ in $10 \mathrm{ml}$ vials, as well as introduction of a 'ready-reckoner' reference chart for prescribing, preparation and administration inserted into each BNFC across the hospital. In September 2018, we implemented a new prescribing aide sticker into kardexes in an effort to reduce IV administration errors. We then audited whether these interventions had led to an overall reduction in adverse incidents related to paracetamol.

Results We demonstrated a progressive reduction in adverse incidents over the past four years; it has now been more than 1000 days since the last reported serious adverse incident related to paracetamol. A snapshot audit of prescribing showed that $100 \%$ of patients were prescribed a safe dose of paracetamol by either age or weight. There was however considerable variation in dosing for enteral prescribing (8.2-18.8 $\mathrm{mg} / \mathrm{kg}$ ), with the majority of children (74\%) being dosed according to age, despite weight being available at time of prescription. Introduction of the paracetamol prescribing aide sticker received overwhelmingly positive feedback from both medical and nursing staff, and has now been implemented for all children requiring regular IV paracetamol.

Conclusions Anaesthetics were the most commonly implicated specialty in paracetamol errors in our institution. Enteral dosing by age banding in hospital carries the potential for some children to be significantly under-dosed leading to sub-therapeutic drug levels, or even inadvertently over-dosed for more complex children who are underweight for age. We would therefore recommend dosing of enteral paracetamol by weight, when available, in hospital. Substantial improvement in paracetamol safety can be achieved through implementation of prescribing and administration aides to reduce likelihood of human error.

\section{REFERENCES}

1. Eyers S, Fingleton J, Perrin K, Beasley R. Proposed MHRA changes to UK children's paracetamol dosing recommendations: modelling study. I $R$ Soc Med 2012:105:263-9.

2. Kaufmann J, Laschat M, Wappler F. Medication Errors in Paediatric Emergencies: A Systematic Analysis. Dtsch Arzteb/ Int 2012;109:609-616. 